
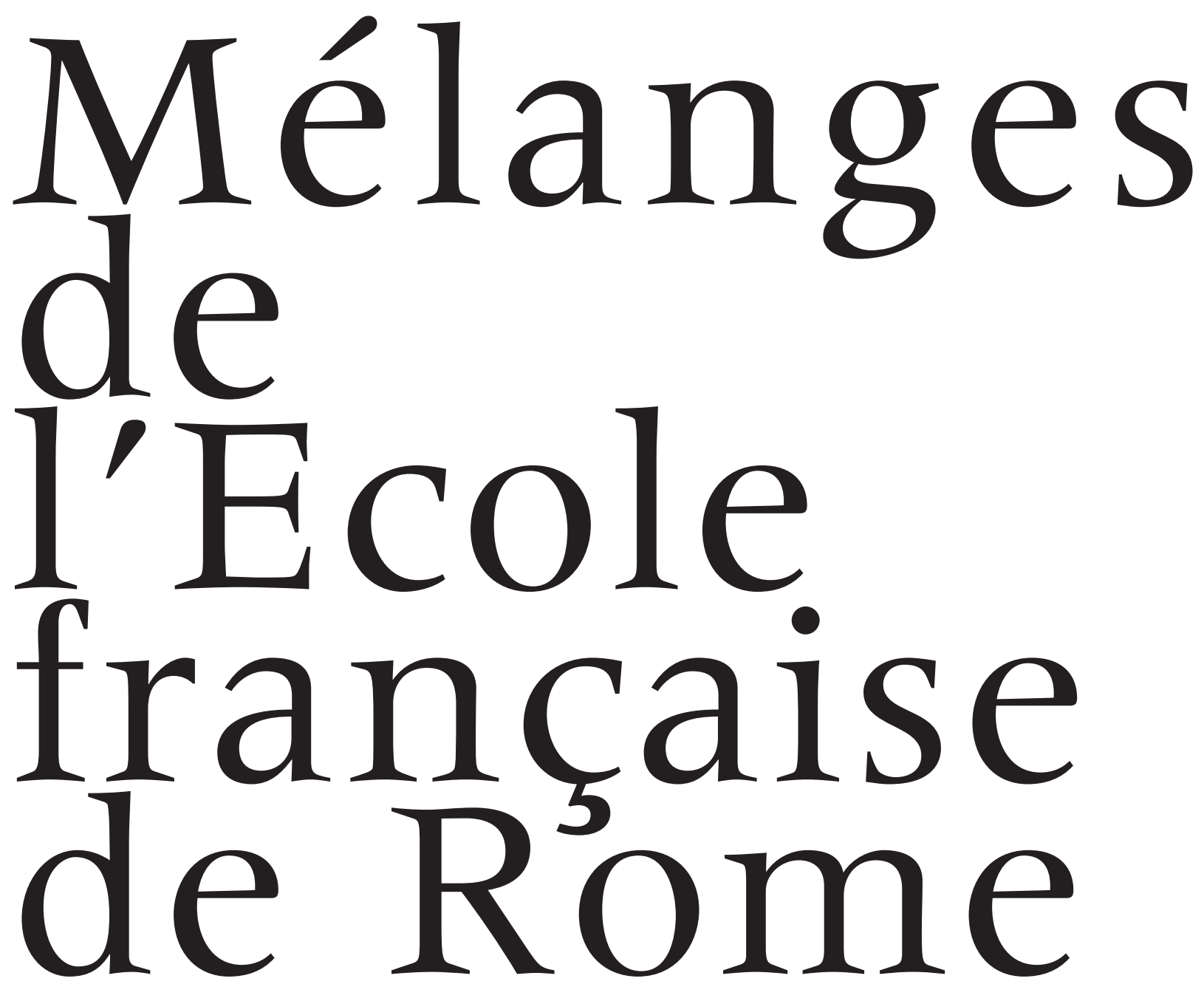

Antiquité

MEFRA

123-1 2011 


\title{
Due divinità di Falerii Veteres : Giunone Curite e Minerva Capta
}

\author{
Giorgio FERRI
}

Il presente studio ha lo scopo di indagare la fisionomia e le caratteristiche di due importanti divinità di Falerii Veteres: Giunone Curite e Minerva Capta. Riprendendo il filo di ricerche già ben sviluppate, si è scelto un peculiare punto di partenza : il rito romano dell'evocatio, nel quale si è ipotizzato più di una volta che le due dee siano potute essere coinvolte; soprattutto per la seconda, come vedremo, ciò pone dei problemi particolari.

Per accennare alle caratteristiche del rito evocatorio, "uno dei capolavori della casistica pontificale ${ }^{1}$, diremo anzitutto che quanto sviluppato dai pontefici costituiva una modalità, se non delle più frequenti, sicuramente tra le più importanti e complesse di "regolazione» del politeismo romano ${ }^{2}$. Il rito era finalizzato essenzialmente all'approccio e all'appropriazione delle divinità, e con esse dei culti, di realtà urbane particolarmente importanti, con il conseguente accrescimento del numero degli dèi presenti a Roma. Tutto ciò avveniva sempre sotto la vigile supervisione e l'efficace azione «filtrante» del collegio dei pontefici, il consesso sacerdotale che aveva specifica competenza in casi di tale rilevanza.

Il processo di accostamento e acquisizione della «nuova» divinità, insieme al suo culto, costituisce un interessante punto di vista su quel sistema religioso romano che, con una definizione solo appa-

1. Bouché-Leclercq 1907, p. 573. Il classico sull'argomento è Basanoff 1947. Da ultimo cfr., anche per tutta la bibliografia precedente, Ferri 2010a; Ferri 2010b.

2. Montanari 2008, p. 11.

3. Famoso esempio è il discorso dell'imperatore Claudio in favore dell'ingresso in Senato di alcuni primores della Gallia rentemente ossimorica e paradossale, potremmo definire allo stesso tempo "fisso" e "fluido"; ciò poiché, sotto l'egida della tradizione costituita dal mos maiorum, si dava una costante capacità di innovare e di adattarsi alle nuove situazioni ${ }^{3}$. Il mos maiorum infatti «è ben più di un semplice indicatore di invarianza. È un concetto dinamico, che si esprime sia come veicolo di trasmissione di valori, sia come segno dell'identità quiritaria, sia come filtro dei processi acculturativi ${ }^{4}$.

Nel caso della celebrazione di un'evocatio, dopo aver "chiamato fuori» la divinità poliade, cui il rito era specificamente riservato, si procedeva dunque all'istituzione del culto a Roma. Già la scelta del sito destinata ad accogliere il tempio di talune divinità evocate ${ }^{5}$ - un esempio su tutti quello della Giunone Regina proveniente da Veio - dice molto sullo status di esse : l'Aventino, al di fuori del pomerium, entro il quale dimoravano le divinità romane o quantomeno italiche ${ }^{6}$. Accoglimento "condizionato", pertanto, il quale, nel momento in cui riconosceva la sostanziale similarità della figura divina evocata con un'altra già presente nel pantheon romano, pur ne sottolineava e ne fissava la "diversità " e l' «alterità», ubicandone il santuario nel luogo "altro" per eccellenza di Roma, al di fuori del confine sacro della città (la situazione muterà solo in età imperiale per iniziativa dell'imperatore Claudio $)^{7}$.

L'importazione di peregrina sacra era conside-

Comata : cfr. CIL XIII $1668=$ ILS 212; Tac. Ann. XI 24, 7; Giardina 1997.

4. Montanari 2008, p. 10.

5. Per tutti i casi, cfr. Ferri 2010a; Ferri 2010b.

6. Catalano 1978, p. 543-544.

7. CIL VI $930=$ ILS 244; Gell. N. A. XIII 14, 4 . 
rata fatto non eccezionale, ma anzi caratteristico del mos maiorum. Essa era effettuata principalmente secondo due modalità : l'evocatio appunto e la consultazione dei libri Sibyllini $i^{8}$. In entrambe tuttavia il compito di "interpretare» in senso romano la divinità portata a Roma, insieme al culto di essa (la cosiddettà interpretatio Romana) ${ }^{9}$ era sempre e comunque appannaggio dei pontefici ${ }^{10}$. Tra i due processi di assunzione di nuovi culti l'evocatio era certamente meno frequente, ed usato solo nei confronti di città dallo status di "capitali", in senso politico, etnico o federale : una delle città avente questa caratteristica è senz'altro Falerii Veteres, l'antica capitale dei Falisci, alla quale ora ci rivolgeremo.

\section{FALERII VETERES}

Non vi è più alcun dubbio sul fatto che l'insediamento di Falerii Veteres vada identificato con l'odierna Civita Castellana ${ }^{11}$, sebbene il nome con cui ci riferiamo all'abitato antico sia in realtà una creazione degli studiosi moderni e non trovi riscontro nelle fonti antiche, in cui compaiono diversi toponimi ${ }^{12}$; stesso discorso quanto a Falerii Novi. Useremo dunque i nomi invalsi negli studi recenti solo per comodità, identificando ovviamente la città antica con Falerii Veteres e la nuova con Falerii Novi.

Il sito di Civita Castellana è posto su di uno sperone tufaceo che si innalza sulle valli del Treia e degli altri corsi d'acqua che in esso confluiscono in quest'area. L'aspetto geomorfologico del colle è in linea con le caratteristiche topografiche della maggior parte degli insediamenti sia del Bronzo Medio e Recente, sia e soprattutto del Bronzo

8. Paul. Fest. 268 L. Per tutte le problematiche relative ai libri Sibyllini, cfr. Santi 2008.

9. Cfr. Ferri 2010a, p. 64-69.

10. Liv. I 20, 6.

11. Cfr. in generale Corretti 1987; Moscati 1990, anche e soprattutto per la storia dell'insediamento urbano; da ultimo Opitz 2009.

12. Cfr. Corretti 1987, p. 324, 331 s., anche sulla storia della ricerca archeologica.

13. Moscati 1990, p. 141-142, 153-154.

14. Paul. Fest. $81 \mathrm{~L}$ : «La città di Faleri trae il suo nome da fale»; Paul. Fest. 78 L : «le falae sono così dette dall'altezza, da falado, che presso gli Etruschi significa cielo", e poco più avanti, s. v. falarica : "da falae, vale a dire luoghi edificati». Cfr. Plut. Cam. 9, 2; 10, 1; Basanoff 1947, p. 97 s.
Finale relativi all'Etruria meridionale : oltre alla presenza di acque perenni, quindi, collocazione su alture ben difese naturalmente e munite di difese artificiali nei punti potenzialmente più vulnerabili ${ }^{13}$. L'imprendibilità della città è forse riflessa nell'etimologia che fa derivare il toponimo da fale, col significato di oppidum ${ }^{14}$; inoltre fala aveva anche il significato di «torre $»^{15}$.

\section{GIUNONE CURITE}

\section{L'epiteto della dea}

Come accadeva in altre città dell'Etruria e del Latium Vetus (ad es. a Veio con Giunone Regina o a Lanuvio con Giunone Sospita), anche la protettrice di Falerii era (una) Giunone ${ }^{16}$, sul cui epiteto tuttavia non vi è accordo nelle fonti. Troviamo infatti : Curitis, Curritis, Quiritis e Curis ${ }^{17}$. Tale varietà sembra riflettere la molteplicità delle funzioni attribuite dalle fonti alla dea, come è possibile d'altronde constatare ogni qual volta si tratti di Giunone ${ }^{18}$; divinità poliade, dunque, ma anche guerriera e con evidenti legami con la sfera femminile : "le matrone si trovano sotto la tutela di Giunone Curite» ${ }^{19}$. Più varianti dell'epiclesi significano più etimologie, proposte sia dagli antichi che dai moderni, ovvero: Cures, curis, currus, quirites e curia ${ }^{20}$.

Radke, così come Dumézil ${ }^{21}$, paragona Iuno Curitis alla Iuno Sospita Mater Regina di Lanuvium; tuttavia, dopo aver rilevato le possibili somiglianze esteriori - l'iconografia le ritraeva entrambe armate di scudo e lancia ${ }^{22}$ - ipotizza un'affinità più profonda a livello semantico : considerato che i nomi terminanti in -tis si riferiscono spesso ad

15. Serv. Ad Aen. IX 705.

16. CIL XI 3100; 3125; 3126. Solo Basanoff 1947, p. 106, ipotizza essere stata (anche) una Fales.

17. Cfr. Aust 1901; Giannelli 1942, p. 218-219, 226; Eisenhut 1963; Dury-Moayers, Renard 1981, p. 161-165; Corretti 1987, p. 326.

18. In generale su Giunone, cfr. Dury-Moayers, Renard 1981; Ferri 2010 b, p. 51-58.

19. Paul. Fest. 55 L.

20. Fonti e bibliografia in Dury-Moayers, Renard 1981, p. 162-165.

21. Dumézil 1954, p. 117.

22. Sul «tipo», cfr. La Rocca 1990, p. 819-822; cfr. DuryMoyaers 1984. 
abstracta, e verificato il significato di "soccorso, aiuto" per l'epiteto Sispita, lo studioso tedesco fa derivare Curitis (*cursitis) da *cursire, *cursis, quindi currere, inteso come "aiutare, correre in aiuto", dunque con il medesimo significato riferibile alla dea di Lanuvio ${ }^{23}$.

Vi è poi l'etimologia riferibile alla città sabina di Cures, come si legge ad esempio in uno scolio a Persio (IV 26) : "A Cures: da tale toponimo Giunone è detta anche Curite poiché colà è venerata con particolare fervore». Ciò ha portato tra l'altro il Basanoff ad ipotizzare che la dea, non appartenente al pantheon locale, sia stata ivi importata e la sua unicità in territorio sabino abbia fatto sì che assumesse l'epiclesi di Curitis $^{24}$. Il culto sarebbe poi stato importato di lì anche a Falerii. Tuttavia, questa ipotesi presuppone una «sabinità» originaria della città da non dare invece per scontata.

Già in antico infatti vi erano posizioni discordanti circa l'appartenenza etnica dei Falisci : oltre alla supposta provenienza da Argo, su cui torneremo, essi sono detti anche duo Etruriae populi (insieme ai Capenati) ${ }^{25}$, quasi certamente per gli

23. Radke 1965, p. 102.

24. Basanoff 1941, p. 126-127; ma cfr. Eisenhut 1963, col. 1325.

25. Plin. $N$. $h$. III 51, narrando fatti accaduti durante l'assedio di Veio.

26. Falisci e Capenati potevano occasionalmente partecipare anche alle riunioni dei populi Etruschi ad fanum Voltumnae : Liv. V 16, 6.

27. Diod. XIV 96, 5 .

28. Cfr. Pallottino $1984^{7}$, p. 275 s.; Corretti 1987, p. 342-344.

29. Varr. ap. Dion. Hal. II 48, 4.

30. Paul. Fest. 56 L; cfr. ibid. 43 L; 55 L.

31. Tert. Apol. XXIV 8. Cfr. Basanoff 1947, p. 107 s.; Eisenhut 1963, col. 1330.

32. Dion. Hal. II 50; cfr. Evans 1939, p. 217 s.; Palmer 1970, p. 152 s.; Palmer 1974, p. 5.

33. Paul. Fest. 56 L; Mart. Cap. II 149.

34. Paul. Fest. 56 L; Dion. Hal. II 50, 3.

35. Palmer 1970, p. 180-181.

36. Ciò per una serie di considerazioni, che esporremo brevemente qui di seguito. Alla stessa organizzazione "curiata" appartenevano anche i Fornacalia, la festa della torrefazione del farro, elemento di capitale importanza economicoreligiosa ai primordi di Roma : "feste della torrefazione del farro" (Plin. N. h. XVIII 8. Cfr. Brelich 20103, p. 159 s.; Sabbatucci 1988, p. 60-63). Anch'essa era festeggiata dalle singole curie, ciascuna in una propria giornata $\mathrm{e}$ in un proprio spazio nel Foro, e non poteva spingersi oltre il 17 febbraio, giorno dei Quirinalia. Il dio Quirino era in stretta relazione con le curie : il suo nome (quirinus da *co-virinus) è una forma aggettivale derivata da curia (*co-viria) (In generale su Quirino e le problematiche ad esso legate, Brelich 1960; Koch 1960; Radke 1981; Sabbatucci 1988, p. 63 s.; Dumézil $2001^{2}$, p. 224-245). Siamo quindi in un contesto stretti vincoli con Veio ${ }^{26}$, oppure entità etnica a sé stante $^{27}$. La posizione attuale rileva gli indubbi caratteri di originalità della civiltà falisca, inserendoli tuttavia in un contesto di influenze esterne soprattutto etrusche ${ }^{28}$.

Varrone riporta l'etimologia a curis, la parola sabina usata per designare l'hasta, la lancia ${ }^{29}$; anche in Festo Curis appare con frequenza come l'appellativo riferito alla dea : "a Giunone, detta Curite ${ }^{30}$. In età tarda, invece, Tertulliano afferma che l'epiteto sarebbe derivato da un non meglio identificato Pater Curris $^{31}$.

Dionigi di Alicarnasso attribuisce a Tito Tazio il culto di una Iuno Curis o Quiris in ognuna delle trenta curiae create da Romolo ${ }^{32}$. Del culto facevano parte l'offerta di acqua e vino ${ }^{33}$ e il sacrificio su mensae curiales, una per ogni curia : "Mensae curiales, nelle quali si sacrificava a Giunone, chiamata Curis" ${ }^{34}$. Il Palmer interpreta la presenza di queste trenta Giunoni come il residuo di una molteplice evocatio avvenuta al momento dell'istituzione del sistema curiato $^{35}$. Tale ipotesi appare comunque inverosimile ${ }^{36}$.

Ma giova tornare subito alla dea oggetto della

cultuale riferibile ad una fase ancora gentilizia della storia di Roma (Sabbatucci 1988, p. 65). Non a caso, nel medesimo periodo si celebravano anche i Parentalia, l'unica festa dei morti dedicata esplicitamente agli antenati (parentes) (cfr. Sabbatucci 1988, p. 48). Tutto ciò per dire che le Iunones Quirites, molto probabilmente, ricevettero il loro nome e l'incarico di tutelare le singole curie all'atto di nascita delle stesse o poco dopo, esclusivamente da ciò traendo la ragione della loro "creazione», senza che vi sia alcun bisogno di ipotizzare una loro evocatio da altri luoghi. Contro questa considerazione urta anche il fatto che l'epiclesi è attestata sia a Falerii che a Beneventum (CIL XI 3125; IX 1547). Inoltre, sarebbe strano che, pur provenendo da luoghi diversi, nessuna di queste Giunoni avrebbe conservato nel nome una nota distintiva «locale». Infine, così come le none di ottobre riguardano le curie, lo stesso avviene alle calende dello stesso mese, in cui si celebrava il culto del cosiddetto Tigillum Sororium, dedicato a Iuno Sororia e Ianus Curiatius (Dion. Hal. III 22, 7-9; Fest. 380 L; Paul. Fest. 399 L; Sabbatucci 1988, p. 318 s.). Se pertanto in questo giorno vi sembra essere una divisione di competenze tra le due divinità, il 7 ottobre esse si riuniscono probabilmente in una sola figura, Iuno Quiritis, protettrice dunque dei viri riuniti (co-viri) in curie (co-virie), ma, per le sue caratteristiche peculiari, con una speciale protezione per la parte delle popolazione da cui lo Stato poteva e doveva attendersi la propria sicurezza e accrescimento, i iuvenes. Ciò potrebbe costituire pure un riflesso cultuale della collatio civitatis tra Romani ed Albani a seguito della contesa tra Orazi e Curiazi, ma anche della realizzazione del novus ordo voluto da Tullo Ostilio, ottenuto creando la Curia Hostilia, dinanzi alla quale le curiae veteres perderanno la loro importanza, che rimarrà solo a livello cultuale. Cfr. Varr. De l. L. V 155: «Le curie erano di due 
nostra trattazione. In proposito, useremo per comodità l'epiteto di Curitis/Curite poiché è quello ritenuto più plausibile dagli studiosi moderni, ferma restando la difficoltà di potersi pronunciare con certezza sulla questione ${ }^{37}$. È certo comunque che le diverse forme fossero equivalenti per i Romani, come risulta evidente dai Fasti, nei quali al 7 ottobre troviamo sia Iovi fulguri [I]unoni Curriti in campo, sia Iovi fulg(uri) Iunoni Q(uiriti) in camp(o) ${ }^{38}$.

\section{Il culto}

Non possediamo alcun documento figurato con l'iconografia della dea ${ }^{39}$. Tuttavia abbiamo delle testimonianze scritte, dalle quali apprendiamo che la dea era ritratta con la lancia in mano : «la chiamavano Giunone Curite poiché ritenevano che portasse la lancia " ${ }^{40}$; di più, lo si è visto, l'hasta era detta in sabino curis : "[Giunone Curite]... così chiamata poiché porta la lancia, detta curis nella lingua dei Sabini ${ }^{41}$. L'aspetto guerriero risulterebbe accentuato inoltre accettandone l'identità con la Curitis di Tivoli - città che, analogamente alla capitale dei Falisci, vantava origini $\operatorname{argive}^{42}$ rappresentata con lo scudo e su un currus $^{43}$. Si veda anche la notizia di Servio : «è detta Curite, quella [Giunone] che fa uso del carro e della lancia ${ }^{44}$.

È invece ormai un dato acquisito identificare il Tempio di Giunone Curite con quello portato alla luce nel 1886 dal Cozza, il cui scavo fu pubblicato $\mathrm{l}^{\prime}$ anno successivo dal Pasqui ${ }^{45}$. Esso è sito in località Celle, su di un altopiano separato da una gola, scavata dal Rio Maggiore, dalla collina di Vignale, in cui si sviluppò l'abitato più antico di Falerii Veteres. Purtroppo il pessimo stato di conserva-

generi : v'erano quelle in cui i sacerdoti curavano le questioni relative agli dèi, come le Curiae Veteres [le Curie antiche] e quelle in cui il Senato curava gli affari riguardanti gli uomini, come la Curia Ostilia».

37. Giannelli 1942, p. 218-219; Eisenhut 1963, col. 1324.

38. Lo stesso sembra accadere a Falerii : cfr. CIL XI 3100; 3126.

39. Per le proposte di identificazione in rilievi, monete e sculture, cfr. La Rocca 1990, p. 835.

40. Paul. Fest. 43 L.

41. Paul. Fest. 55 L.

42. Cfr. infra.

43. Cfr. Eisenhut 1963, col. 1331; La Rocca 1990, p. 836.

44. Serv. Ad Aen. I 8; cfr. ibid. I 17; IV 59.

45. Pasqui 1887. In generale sul tempio, Basanoff 1941, p. 111 s.; Comella 1986, p. 177.

46. Sulla pianta, cfr. Comella 1986

47. Lo stesso sistema di cunicoli, vasche e cisterne è osservabile nei due santuari posti sull'altura di Vignale : cfr. Moscati zione, dovuto anche alla secolare azione distruttrice del corso d'acqua prospiciente la parte anteriore dell'edificio, ha generato più di una discussione circa l'esatta fisionomia della pianta, di volta in volta considerata a tre celle, ovvero ad una cella con alae laterali ${ }^{46}$.

Quel che è certo, tuttavia, è che il santuario avesse dimensioni grandiose, degne della divinità poliade della capitale di una realtà politica di non trascurabile importanza quale la falisca. Inoltre, in conformità con la natura geologica dei luoghi dell'Etruria Meridionale - frequenti le formazioni tufacee di origine vulcanica - e l'uso di origine etrusca di incanalare le acque per raccoglierle e conservarle, nel tempio di Celle si osserva la presenza di un bacino rettangolare di fronte alla cosiddetta «abside» e di una vasca davanti all'edificio $^{47}$. Entrambi erano alimentati da cunicula e vi sono stati rinvenuti numerosi materiali votivi, tra cui degli ex voto anatomici ${ }^{48}$. L'acqua costituiva dunque una parte integrante del complesso cultuale, ed era usata verosimilmente a fini lustrali e salutari ${ }^{49}$. Lo stesso avveniva, ad esempio, a Veio nel tempio di Portonaccio e presumibilmente in quello di Giunone Regina ${ }^{50}$, ma anche a Volsinii nel tempio della "Venere di Cannicella ${ }^{51}$.

Per quel che riguarda più propriamente il culto, abbiamo un'interessante testimonianza di Dionigi di Alicarnasso, dal quale risulta evidente che esso riguardasse particolarmente le donne, in veste di sacerdotesse ${ }^{52}$. Ancor più notevole è quanto tramanda Ovidio, che assistette in prima persona al rito celebrato in onore della dea nel suo tempio presso Falerii, città natale della moglie ${ }^{53}$. Dal poeta di Sulmona apprendiamo che esso

1990 , p. 155 s

48. Comella 1986, p. $181 \mathrm{~s}$

49. Plin. N. h. II 230; cfr. Basanoff 1941, p. 117, 132 s.

50. Cfr. Ferri 2010a, cap. IV.

51. Basanoff 1947, p. 76-77; Stopponi 1985.

52. Dion. Hal. I 20-21.

53. Am. III 14 : «Essendo mia moglie nata nella fruttifera terra di Faleri, raggiungemmo le mura vinte da te, o Camillo. Le sacerdotesse preparavano la casta festività di Giunone e giochi molto frequentati e il sacrificio di una giovenca del luogo. Conoscere quel rituale è abbondante ricompensa alla sosta, anche se la strada verso là presenta un percorso difficile per le salite. Vi è un bosco sacro vecchio e oscuro per la densità degli alberi; guardalo : ammetterai che è sede di divinità. Accoglie le preghiere e l'incenso votivo dei fedeli un altare, un altare fatto senza arte da mani antiche. Di qui, non appena il flauto ha cominciato a suonare con le sue solenni note si snoda per le strade ornate la processione annuale. 
comprendeva giochi solenni, una processione composta da giovani e giovinette (che cantavano anche degli inni) ${ }^{54}$, dalle sacerdotesse del culto e ovviamente dal simulacro della divinità. Vi era poi il sacrificio di giovenche bianche, di un maiale e di un ariete, oltre ad un rito più cruento in cui si cacciava una capra, che andava in premio al giovane che riusciva a ferirla ${ }^{55}$. Il rituale era forse avviato da una fanciulla nubile detta kanephoros ${ }^{56}$, ma esisteva anche un pontifex sacrarius Iunonis Curritis $^{57}$. A partire dalla descrizione ovidiana, si è identificato il luogo di culto da cui prendeva le mosse la processione nell'area in fondo al Fosso dei Cappuccini, in cui infatti si trovano un altare, una vasca e tracce di una strada abbastanza impervia che conduce al santuario di Celle ${ }^{58}$.

\section{La probabile evocatio del 241 a.C.}

Da questo tempio fu verosimilmente evocata la dea. In proposito, è opportuno accennare allo svolgimento dei fatti storici. La capitale dello stato falisco fu assediata ed espugnata una prima volta da Camillo nel 394 a.C., in ritorsione degli aiuti portati a Veio per tutta la durata dell'assedio ${ }^{59}$, conclusosi solo due anni prima con la conquista e il saccheggio della potente rivale ${ }^{60}$. Ma le operazioni non durarono a lungo : nelle fonti l'evento risolutivo è attribuito, con poca verosimiglianza storica, al noto episodio del tradimento del

Vengono condotte, fra l'applauso del popolo, giovenche bianche come la neve che l'erba dei Falisci ha nutrito nei suoi campi, e vitelli non ancora minacciosi con la fronte che fa temere e, vittima più piccola da un modesto porcile, un porcellino e il capo del gregge con le corna ricurve sulle dure tempie; solo la capretta è detestata dalla dea potente : si dice che per delazione di questa fu scoperta nel profondo bosco e dovette abbandonare la fuga intrapresa. Ancora oggi la delatrice è attaccata con giavellotti dai ragazzi e viene data come premio a colui che la ferisce. Per dove la dea passerà, giovani e timide fanciulle scopano le ampie vie e vi stendono tappeti. I capelli delle sacerdotesse sono caricati d'oro e di pietre preziose, e una magnifica veste copre fino ai piedi ornati d'oro; secondo il costume greco dei padri, coperte con abiti bianchi portano sulla testa gli oggetti sacri loro affidati. Stanno in silenzio le genti allora quando giunge la processione ricca d'oro e la dea segue le sue sacerdotesse. Argivo è l'aspetto della processione: ucciso Agamennone, Aleso cercò di sfuggire al delitto e alle ricchezze della patria e profugo, dopo aver errato per terra e per mare, fondò con mano fortunata le nobili mura. Egli insegnò ai suoi Falisci le cerimonie a Giunone: esse siano sempre propizie a me, sempre al suo popolo!n

54. Cfr. Dion. Hal. I 21, 2.

55. Cfr. Eisenhut 1963, col. 1329-1330. maestro di Falerii ${ }^{61}$, episodio che potrebbe adombrare o costituire una ripetizione della deditio di un secolo e mezzo dopo ${ }^{62}$.

Vi furono poi altri scontri tra Falisci e Romani, intervallati da foedera e paci, fino alla tappa fondamentale costituita dalla conquista e distruzione della città da parte dei Romani nel 241 a.C., poiché nell'ultimo anno della prima guerra punica i Falisci si erano ribellati al giogo di Roma ${ }^{63}$. Concluse le ostilità con Cartagine, furono inviati in territorio falisco due eserciti consolari che ebbero ragione dei rivoltosi in sei giorni ${ }^{64}$. La città fu presa d'assedio, che comunque non durò a lungo visto che i Falisci si arresero presto, dandosi in fidem, atto che molto probabilmente evitò l'asservimento, se non il massacro della popolazione $^{65}$. La resa fu accettata e i termini imposti furono la consegna delle armi, dei cavalli, degli schiavi e di oltre metà del territorio, annesso in qualità di ager publicus ${ }^{66}$. Inoltre, la popolazione fu costretta a trasferirsi in un nuovo insediamento a circa $6 \mathrm{~km}$ dall'oppidum primitivo, Falerii Novi, i cui resti sono tuttora visibili, in primis la pregevole porta ad arco, perfettamente conservata, presso l'attuale località di S. Maria in Falleri ${ }^{67}$. I consoli Quinto Lutazio Cercone e Aulo Manlio Torquato Attico celebrarono all'inizio del marzo 240 il trionfo $^{68}$, regolarmente riportato dai Fasti ${ }^{69}$, ma di cui possediamo anche una testimonianza più singolare : un'iscrizione datatoria apposta sulla

56. Dion. Hal. I 21, 2.

57. CIL XI 3125; 3100

58. Comella 1986, p. 185.

59. Cfr. Liv. V, 8-19; Plut. Cam. 2, 10.

60. Liv. V 26-27.

61. Liv. V 27; Plut. Cam. 9-10. Dumézil ha paragonato l'episodio sia con il mito vedico dell'Aurora sia con il rito romano dei Matralia : cfr. Dumézil 1980, p. 93-102.

62. Cfr. Liv. V 27, 12 : "vinti da voi e dal vostro comandante ci arrendiamo a voi...".

63. In generale sul conflitto, le cause e gli eventi successivi, cfr. Loreto 1989; Di Stefano Manzella 1990.

64. Liv. Per. 20; Eutr. II 28.

65. Val. Max. VI 5, 2; cfr. Loreto 1989, p. 723; Di Stefano Manzella 1990, p. 344.

66. Cfr. Di Stefano Manzella 1990, p. 345.

67. Zonar. VIII 18. Sullo status giuridico della nuova fondazione e la composizione dei suoi abitanti, cfr. Di Stefano Manzella 1990. Non si comprende la motivazione che porta Bernardi 1979, p. 98, ad ubicare il nuovo abitato nell'odierna Civita Castellana, in realtà sede dell'antico.

68. Sui problemi relativi alla cronologia, cfr. Loreto 1989, p. 719720; sull'eccezionalità del trionfo celebrato da entrambi i consoli, ibid., p. 728 s.

69. InscrIt XIII.1, 549. 
parte anteriore di una corazza bronzea da cavaliere facente parte del bottino di guerra di un soldato romano ${ }^{70}$.

$\mathrm{Fu}$ praticata un'evocatio prima dell'espugnazione della capitale falisca? Purtroppo non vi sono testimonianze in proposito, ma la cosa è in sé probabile. Anzitutto vi è il contesto bellico : Falerii dapprima si rivoltò, poi fu cinta d'assedio ${ }^{71}$. L'essersi consegnata in fidem non evitò la distruzione della città $^{72}$ (ma i templi più importanti furono risparmiati), e forse a questa decisione contribuì, oltre alla prospettiva di finire in catene $o$ peggio, la diffusione della notizia della celebrazione del rito dinanzi alle mura, segno tangibile che gli dèi locali erano passati dalla parte dei Romani ${ }^{73}$.

Vi era d'altronde un parallelo molto indicativo, vicino nel tempo e nello spazio : la conquista e distruzione di Volsinii nel 265 a.C., dunque solo 24 anni prima e a non molta distanza dalla capitale falisca. La caduta della città etrusca, centro federale e religioso del nomen, doveva aver destato molto scalpore nelle menti dei Falisci, alleati storici degli Etruschi, in particolare di Veio. Al termine di quel conflitto era stato verosimilmente evocato Voltumna $^{74}$.

Analogamente, non si doveva ignorare neanche la sorte di Veio, messa a ferro e fuoco dopo essere stata privata della sua Giunone. Inoltre, così come Veio (all'epoca dell'assedio la città etrusca più potente), Volsinii e poi Cartagine, Falerii era la capitale di un'entità statale, per cui forse si considerò necessario procedere alla celebrazione di un'evocatio.

\section{Il culto successivo a Roma e a Falerii}

Valga anche la considerazione che pure Giunone Curite ricevette un tempio a Roma, nel Campo Marzio : Iovi Fulguri Iunoni Quiriti in campo

70. Zimmermann 1986. Il testo recita : Q. Lutatio. C. F. A. Manlio. C. F. I Consolibus. Faleries. Capto. Il pezzo si trova al Getty Museum di Malibu.

71. L'evocatio era infatti praticata in obpugnationibus : Liv. V 21 , 1-3; Plin. N. h. XXVIII 18; Fest. 268 L; Serv. Ad Aen. II 244; Macr. Sat. III 9, 2.

72. Loreto 1989, p. 722; Di Stefano Manzella 1990, p. 365.

73. Cfr. tuttavia Macr. Sat. III 9, 2 sull'evocatio come pratica segreta e sconosciuta a molti.

74. Cfr. Ferri 2009

75. Basanoff 1941, p. 136 s., ritiene che il testo mancante del calendario precesareo di Anzio al $1^{\circ}$ giugno vada così integrato: IUNONI. IN. [CAMPO.], supponendo dunque che vi è l'annotazione in vari calendari al 7 ottobre $^{75}$. Ci troviamo dunque al di fuori del pomerio, dal che possiamo almeno supporre che la divinità fosse considerata straniera ${ }^{76}$.

Se è certa l'ubicazione del santuario nella zona del Campo Marzio, molto più incerta è l'identificazione del luogo preciso ed eventualmente il riconoscimento in strutture ancora conservate: Castagnoli lo aveva riconosciuto nel Tempio A dell'area sacra di Largo Argentina ${ }^{77}$, ma tale ipotesi è stata rifiutata da Coarelli ${ }^{78}$ e Ziolkowski, il quale a sua volta aveva proposto come possibili resti del tempio quelli trovati nel 1877 sotto la demolita chiesa di S. Anna ${ }^{79}$. Basanoff, dal canto suo, propende per il Tempio C di Largo Argentina, ai margini della Palus Caprae ${ }^{80}$. La recente ipotesi del Manacorda, invece, prospetta la possibilità di identificare il tempio in uno dei due edifici rappresentati nella Forma urbis Romae del Lanciani (fr. 234b-c) ${ }^{81}$, e di collocarlo in stretta relazione con i templi di Vulcano e Iuppiter Fulgur, subito a S dell'area sacra di Largo Argentina e a O della cavea del theatrum Balbi ${ }^{82}$. Pur con alcune differenze nella sistemazione della topografia del luogo, anche Coarelli ritiene di localizzare il tempio di Iuno Curitis alle spalle del Teatro di Balbo, sempre in stretta connessione con il tempio di Iuppiter Fulgur : entrambi gli dèi sarebbero stati evocati da Falerii $^{83}$.

Nonostante il più che probabile trasferimento del simulacro a Roma, il culto della dea nel suo santuario di Celle non cessò. La presenza di ex voto databili alla seconda metà del III e al II sec. a.C., e, in modo più esauriente, le terrecotte architettoniche confermano la continuità del culto ${ }^{84}$, anche se più modesto che in precedenza. Vi è anche un'iscrizione di età imperiale che fa cenno al lucus Iunonis Curitis $^{85}$. Non solo, ma, come abbiamo visto

fosse un'altra festività dedicata a Iuno Curitis, risalente peraltro ad una fase precedente al conflitto con Falerii.

76. Cfr. Catalano 1978, p. 543-544; Ferri 2009, p. 1000.

77. Castagnoli 1946, p. 169-175

78. Coarelli 1981, p. 43; Coarelli 2003³, p. 338.

79. Ziolkowski 1992.

80. Basanoff 1941, p. 139 s

81. Lanciani 1990-2007, tav. XXI.

82. Manacorda 1999, p. 121.

83. Coarelli 1997, p. $211 \mathrm{~s}$

84. Comella 1986, p. 184

85. CIL XI 3126. 
poco sopra, ogni anno si celebrava presso il tempio della dea il sontuoso rito cui assistette personalmente Ovidio. Anche il sito del primitivo insediamento continuò ad essere popolato, come testimoniano i ritrovamenti, anche se naturalmente la contrazione dell'abitato fu notevole ${ }^{86}$.

Le ipotesi circa il destino dei culti di Falerii dopo la distruzione della città variano considerevolmente. Hülsen ritiene che i Romani abbiano lasciato il culto e il tempio di Iuno Curitis sul posto, e abbiano invece portato a Roma i culti di Giano e Minerva ${ }^{87}$. Wissowa è invece del parere che Iuno Curitis sia stata evocata nel $241^{88}$. Più articolata la posizione del Basanoff : il tempio nel Campo Marzio sarebbe stato dedicato nel 388 a.C. a seguito di un'exoratio (una preghiera solenne), con la quale i Romani importarono il culto della dea e se ne assicurarono il sostegno senza evocarla, mentre l'evocatio vera e propria avrebbe avuto luogo più tardi, con la conquista della città ${ }^{89}$.

\section{MINERVA CAPTA}

\section{Un problema spinoso}

Un problema particolare, legato anche all'evocatio, riguarda la figura di Minerva Capta, giunta a Roma da Falerii nel 241 a.C. assieme a Iuno Curitis e Ianus Quadrifrons ${ }^{90}$. Dando infatti all'epiteto il significato di "catturata", come fanno alcuni studiosi ${ }^{91}$, ci troveremmo di fronte al compito di rispondere alla domanda : era possibile prendere prigioniera una divinità?

86. Moscati 1990, p. 169-170; Di Stefano Manzella 1990, p. 361; contra Loreto 1989, p. 722

87. Hülsen 1909, col. 1970.

88. Wissowa $1912^{2}$, p. 49 e 187.

89. Basanoff 1947, p. $52 \mathrm{s.}$

90. Martin 1987, p. 52, ritiene che anche il Giano falisco abbia subito un'evocatio. Altre divinità molto venerate presso i Falisci erano anche Marte (cfr. Ov. Fast. III 89; Liv. XXII 1, 11; Plut. Fab. Max. 2, 2) ed Apollo sul monte Soratte (Verg. Aen. XI 785; Plin. N. h. XIX; Solin. II 26).

91. Ad es. Wissowa 1899; Bruun 1972, p. 120; Montanari 1993, p. 271; Dumézil $2001^{2}$, p. 272,371 .

92. III 835-848 : «Là dove il monte Celio discende dall'alto nel piano, e la via, se non del tutto, v'è press'a poco piana, puoi vedere il piccolo tempio di Minerva Capta, che fu consacrato alla dea nel suo giorno natalizio. L'origine di quel nome è incerta. Capitale definiamo un fervido ingegno : e la dea è ingegnosa per eccellenza. Oppure perché si dice che, priva di madre, balzasse dal capo del padre, già imbracciando il suo
La fonte che ci informa dell'esistenza di questa dea sono i Fasti di Ovidio, che fa derivare il particolare epiteto da caput o da capere ${ }^{92}$. Questa particolare Minerva era stata portata a Roma dopo la conquista della sua città : da ciò dunque il suo particolare status di "prigioniera», forse perché aveva rifiutato di farsi evocare, come pure è stato ipotizzato?

Le ipotesi degli studiosi sono varie e contrastanti : L. Preller ritiene, con altri, che l'epiteto derivi dal fatto che la testa (caput) è la sede del pensiero ${ }^{93}$. Altri invece intendono capta con "presa", "accettata" ${ }^{94}$. G. Radke ritiene che Capta fosse il vero nome e Minerva l'epiteto della divinità ${ }^{95}$. Un'ipotesi interessante è anche quella di T. Köves-Zulauf ${ }^{96}$, che ritiene che alla desinenza -ta sia da attribuire un significato attivo, come accade per altri epiteti ${ }^{97}$ : Capta starebbe di conseguenza per "l'accogliente» ${ }^{98}$. P. E. Huschke deduce dal fatto che nel 241 a.C. Falerii si sia arresa e consegnata in fidem, non in potestatem, che non poteva aver avuto luogo alcuna cattura ${ }^{99}$.

\section{Era possibile prendere prigionieri gli dèi?}

D'altronde, contro la consueta interpretazione di capta $=$ "prigioniera», linguisticamente ineccepibile, contrasta fondamentalmente la considerazione che il concetto di una divinità prigioniera costituirebbe un unicum nella storia antica. Esistono delle rappresentazioni di divinità avvinte come Era, Afrodite e Prometeo, ma in questi casi il dio è imprigionato da un altro dio ${ }^{100}$; anche nel

scudo? O perché dopo aver domato i Falisci venne a noi prigioniera [captiva], come attesta un'antica iscrizione? O perché la legge del suo santuario impone che i furti compiuti in quel luogo si paghino con la pena capitale? O Pallade, da qualunque origine tu tragga quel nome, imbraccia sempre l'ègida in difesa dei nostri prìncipi!». Cfr. LTL, s.v.

93. Preller 1978, p. 260.

94. Ad es. Gustafsson 2000, p. 58.

95. Radke 1965, p. 81.

96. Köves-Zulauf 1993; cfr. Ferri 2006, p. 231-236.

97. (Iuno) Februata, (Mater) Matuta, (Fortuna) Viscata : queste divinità hanno compiuto le azioni inerenti al loro epiteto, le possono sempre compiere e le compiranno: februare $=$ "purificare», *matuere $=$ "fare del bene", *viscare $=$ «invischiare".

98. Cfr. TLL, s. v. capio, p. 330.

99. Huschke 1970, p. 355 s.

100. Cfr. $O d$. VIII, 267 s.; Meuli 1975, p. 1062 s.; Icard-Gianolio 2004, p. 468-471. 
caso dell'imprigionamento di Pico e Fauno da parte di Numa, questi riesce nel suo intento solo conformemente al volere di Giove e con l'aiuto della ninfa Egeria ${ }^{101}$.

Da alcuni testi relativi alla conquista di città, tuttavia, si potrebbe ricevere l'impressione che fosse possibile prendere prigionieri anche gli dèi. Ad esempio, nella formula di deditio la città si dichiara pronta a "consegnare tutte le cose divine ed umane ${ }^{102}$. Molti includono nei divina non solo gli oggetti sacri e i doveri di culto, ma anche gli esseri divini, dato che humana comprende sia le cose che le persone. In secondo luogo, alla presa di una città è spesso associata la rimozione delle statue di culto, con o senza un'evocatio, e si parla anche di di ablati, di dèi "portati via" ${ }^{103}$. Infine, abbiamo precisa menzione di una categoria definita di dèi, gli dei captivi ${ }^{104}$. L'elemento unificante di questi tre argomenti è costituito dalla statua della divinità : la possibilità di prendere prigioniero un dio è legata alla misura in cui si ritiene possano coincidere numen e simulacro ${ }^{105}$.

In nessun caso possiamo però parlare di identità. Solo la statua puo essere presa, e solo con il consenso della divinità : "La statua, solo la statua, può essere catturata, il dio da parte sua si muove di sua iniziativa, libero, insieme al vincitore, quale suo adiutore divino ${ }^{106}$. Macrobio ritiene che per $\mathrm{i}$ Romani fosse sacrilego prendere prigionieri gli dèi ${ }^{107}$ : proprio per questo in realtà questi di captivi non sono mai esistiti. Tertulliano parla di dei captivi intendendo verosimilmente quelli giunti a Roma in seguito a campagne belliche, distinguendoli da quelli "adottati» in tempo di pace.

È da escludere quindi che presso i Romani vi fossero delle divinità prigioniere per definitionem, presso un popolo cioè che vedeva nella sottomis-

101. Ov. Fast. III 261 s.; sull'episodio, cfr. Mora 1994, p. 111-115.

102. Liv. I 38; VII 31,3; XXVI 33, 12 s.; XXVIII 34, 7; cfr. Rüpke 1990, p. 209-210.

103. Liv. XXVI 30, 9; XXVI 33, 12 s.; XXVII 16, 7; Aug. De civ. Dei I 6; Serv. Ad Aen. VII 607

104. Tert. Apol. X 5 : "Or dunque dovrei io passare in rassegna uno per uno tanti e svariati dèi, nuovi e antichi, barbari e greci, romani e forestieri, captivi e adottivi, propri e comuni, maschi e femmine, rustici e urbani, nautici e militari?».

105. Cfr. Van Doren 1954, p. 494-497; in generale Funke 1981

106. Köves-Zulauf 1993, p. 161; cfr. Ferri 2010a, p. 53-56.

107. Macr. Sat. III 9, 2 .

108. Hor. Carm. III 6, 5: "Perché ti riconosci [scil. o Romano] inferiore agli dèi, tu comandi agli altri popoli». L'essenza sione agli dèi il motivo principale del proprio successo $^{108}$. Se fosse stato possibile prendere prigioniero un dio insieme alla sua statua, inoltre, non si vede perché delle decine di divinità "prese» solo una sarebbe dovuta essere caratterizzata dall'epiteto Capta. È più probabile che la Minerva di Falerii avesse già l'epiteto che la caratterizzava. Resta pertanto da capire come essa lo abbia ricevuto.

\section{Un tentativo di interpretazione}

È possibile che Minerva a Falerii potesse essere già da tempo «avvinta» da catene o legami di altra sorta, secondo un uso ben attestato nel mondo greco e nel Vicino Oriente, come ad esempio Artemide ad Efeso, Era a Samo e Apollo a Tiro ${ }^{109}$. Anche a Roma vi era una statua di Saturno legata da corde di lana, che venivano sciolte in occasione dei Saturnalia ${ }^{110}$; Elagabalo incatenò il Palladio nel tempio da lui costruito sul Palatino $^{111}$; la Tyche di Costantinopoli fu rappresentata incatenata ${ }^{112}$.

Le statue di culto erano legate per più motivi : per limitare o quantomeno tenere sotto controllo divinità considerate pericolose; per «liberare» la loro benevolenza in occasione della festa annuale loro dedicata ${ }^{113}$, comprendente spesso anche una processione; oppure, infine per il timore che il dio potesse abbandonare i propri protetti ${ }^{114}$, anche a seguito di un'evocatio ${ }^{115}$ : stesso principio per cui la Nike di Atene era rappresentata senz'ali, a significare che non avrebbe mai dovuto abbandonare la città $^{116}$. Probabilmente furono queste rappresentazioni ad influire sul mito, non viceversa ${ }^{117}$.

Questa concezione giunse a Falerii molto probabilmente attraverso i rapporti con la Magna

della pietas è il riconoscimento dell'assoluta superiorità della natura divina : cfr. Cic. De nat. deor. I 45; ibid. I 56.

109. Su quest'ultimo, cfr. Diod. XVII 41, 7-8; Plut. Q. R. 61. Sull'argomento cfr. in generale Meuli 1975; Icard-Gianolio 2004, p. 468-471.

110. Macr. Sat. I 8, 5; Stat. Silv. I 6, 4; su Saturno, cfr. Brelich $2010^{3}$, p. 113 s.; sui Saturnalia, Sabbatucci 1988, p. 344-355. 111. HA III 4

112. Fonti in Meuli 1975, p. 1081.

113. Meuli 1975, p. 1043.

114. Schol. Pind. Ol. VII 95. Cfr. Ziehen 1949, col. 186.

115. Schwenn 1920-1921, p. 317; Meuli 1975, p. 1077

116. Paus. III 15, 7

117. Schwenn 1920-1921, p. 319. 
Grecia, che certamente vi furono, anche se mediati dagli Etruschi, i quali ebbero costanti ed intense frequentazioni con i $\mathrm{Greci}^{118}$, sia pacifiche, in particolare nel corso della loro presenza in Campania ${ }^{119}$, ma anche per il tramite degli scambi commerciali - ricordiamo ad esempio i rinvenimenti e le influenze osservabili nei porti di Tarquinia e Caere, Gravisca e Pyrgi - sia occasioni di scontro armato, come la battaglia di Cuma del 474 a.C., vinta da Ierone, e il saccheggio di Pyrgi nel 384 a.C. ad opera di Dionigi ${ }^{120}$ : due tiranni di Siracusa dunque, colonia di Corinto ${ }^{121}$.

I rapporti con la città dorica sono intensi in età orientalizzante (VII sec. a.C.) e si riflettono nella storia di Demarato, della famiglia aristocratica corinzia dei Bacchiadi, che, a seguito della presa di potere del tiranno Cipselo (657 a.C.) si sarebbe rifugiato a Tarquinia, imparentandosi poi con la nobiltà locale e avendo un figlio, Lucumone, che sarebbe poi diventato il re di Roma Lucio Tarquinio Prisco ${ }^{122}$. Egli avrebbe insegnato agli Etruschi la scrittura e gli artisti al suo seguito avrebbero introdotto in Italia l'arte plastica ${ }^{123}$.

I Corinzi, i fondatori di Siracusa, appartenevano all'ethnos dorico, lo stesso di Argo. Proprio a questa città peloponnesiaca i Falisci facevano risalire le proprie origini : la città sarebbe stata fondata da un Halaesus/Halesus argivo, a seconda delle fonti considerato ora un compagno, ora un figlio bastardo di Agamennone, oppure figlio di Nettuno $^{124}$.

Più di un autore individuava delle somiglianze tra Argo e Falerii, soprattutto esteriori e in ambito religioso ${ }^{125}$, motivandole con la supposta origine argiva della città falisca; d'altronde nelle fonti è testimoniata con frequenza una diffusione del culto di Hera Argiva, dal Sele fino alla regione dei

118. Cfr. Bonghi Jovino 1986.

119. Pallottino $1984^{7}$, p. 135 s.

120. Cfr. Ibid., p. 202, 235.

121. Sulla storia della colonizzazione greca in Italia cfr. Musti $1999^{8}$, p. 179 s.; Musti 2005.

122. Dion. Hal. III 46 s.; Strab. V 2, 2; Plin. N. h. XXXV 16; cfr. Pallottino $1984^{7}$, p. 180-181.

123. Tac. Ann. XI 14; Plin. N. h. XXXV 152.

124. Fonti in Corretti 1987, p. 323; la tradizione circa l'origine argiva è ben radicata a partire da Catone, e si riflette anche in epoca tarda su Falerii Novi, fondata secondo Solin. II 7 da un Falerius argivo, altrimenti ignoto.

125. Va menzionato anche, in contesto militare, l'uso di armi di tipo "argolico».

126. Dion. Hal I 21, 2; Ov. Am. III 13, 31. Anche la Iuno di Tibur è detta Argeia: CIL XIV 3556. Cfr. La Rocca 1990, p. 815-816.
Veneti ${ }^{126}$. Le analogie erano individuate nell'iconografia della dea, nel culto (sacrificio, funzione delle donne consacrate al culto, presenza di una kanephoros nubile destinata a dare principio al culto, cori di vergini), ma anche nell'aspetto dell'edificio di culto : il tempio era ritenuto simile all'Heraion di Argo, ed era analogamente orientato verso sudest $^{127}$. Vi erano altri santuari di Hera in Magna Grecia considerati «argivi» : alle foci del Sele, non lontano da Poseidonia-Paestum e al capo Lacinio presso Crotone ${ }^{128}$.

Riassumendo, è possibile che Falerii, forse per il tramite etrusco, abbia accolto alcuni influssi del mondo greco, in particolare di ambito dorico, in alcuni usi e nel culto : troppe sono infatti le testimonianze per supporre uno sviluppo indipendente. Tra questi elementi vi dovette essere dunque anche la consuetudine di incatenare la statua di culto per evitare che abbandonasse la città, usanza diffusa sicuramente presso i Dori e le loro colonie, come testimoniano i casi dell'Artemide Orthia Lygodesma di Sparta e dell'Artemide Lyaia di Siracusa ${ }^{129}$.

Sulla scelta di Minerva non possiamo pronunciarci, tranne forse che per la considerazione che la "messa in catene" dovette essere necessariamente successiva rispetto all'adozione di Giunone Curite quale divinità poliade. Infine, possiamo constatare come nella nostra fonte principale, Ovidio, Minerva non sia concepita né rappresentata in catene : egli non avrebbe certo fatto passare sotto silenzio un particolare così inconsueto ${ }^{130}$. Tuttavia, il poeta di Sulmona la vide più di due secoli dopo, nel suo tempietto sul Celio ${ }^{131}$, ovviamente libera da vincoli, necessariamente recisi per far sì che la dea fosse portata a Roma, anche con il grande effetto propagandistico che ciò dovette

127. Dion. Hal. I 21; La Rocca 1990, p. 835.

128. La Rocca 1990, p. 816.

129. Meuli 1975, p. 1044 s.

130. Köves-Zulauf 1993, p. 159. Alcuni hanno proposto un paragone con l'episodio del ratto del Palladium da parte di Ulisse e Diomede, ma in verità in modo poco convincente : tra le discordanze il fatto che nel caso di Atena l'accaduto è un sacrilegio, che scatena l'ira divina e deve portare alla restituzione dell'idolo. Cfr. Canciani 1987.

131. Cfr. Varr. De l. L. V 47 : "Ceriolense : quarto santuario vicino al tempio di Minerva, là dove comincia la salita per andare sul monte Celio". Si è supposto che il sacello fosse posto nell'area della chiesa dei SS. Quattro Coronati : Basanoff 1947, p. 51; Girard 1989, p. 164, che ipotizza inoltre essere stato il 19 marzo il dies natalis del tempio. 
comportare. Togliendo all'epiteto della dea il suo significato negativo, in teoria potremmo ipotizzarne un'evocatio, come taluni hanno fatto ${ }^{132}$, ma contro tale considerazione osta il fatto che la divi- nità poliade della capitale falisca era Giunone Curite : com'è noto, il rito è rivolto specificamente alla divinità tutelare della città ${ }^{133}$.

Giorgio FERRI

\section{Abbreviazioni bibliografiche}

Aust 1901 = E. Aust, Curritis, in RE, IV,2, 1901, col. 18451846.

Basanoff $1941=\mathrm{V}$. Basanoff, Junon falisque et ses cultes à Rome, in Revue de l'Histoire des Religions, 124, 1, 1941, p. 110-141.

Basanoff $1947=$ V. Basanoff, Evocatio : étude d'un rituel militaire romain, Paris, 1947.

Bernardi 1979 = A. Bernardi, Storia d'Italia. Dalla preistoria al principato augusteo, in Storia d'Italia, I, Novara, 1979.

Bonghi Jovino 1986 = M. Bonghi Jovino, Gli Etruschi e la Magna Grecia, in Rasenna, p. 717-723.

Bouché-Leclerq $1907=$ A. Bouché-Leclerq, Pontifices, in C. Daremberg, E. Saglio (a cura di), Dictionnaire des Antiquités, IV, 1, Paris, 1907, p. 567-578.

Brelich $1960=$ A. Brelich, Quirinus. Una divinità romana alla luce della comparazione storica, in SMSR 31, 1960, p. 63-119.

Brelich $2010^{3}=\mathrm{A}$. Brelich, Tre variazioni romane sul tema delle origini, a cura di A. Alessandri, Roma, 2010³.

Bruun 1972 = P. Bruun, Evocatio deorum : some notes on the Romanization of Etruria, in H. Biezais (a cura di), The myth of the state, Stoccolma, 1972, p. 109-120.

Castagnoli 1946 = F. Castagnoli, Il Campo Marzio nell'antichità, Roma, 1946.

Catalano $1978=$ P. Catalano, Aspetti spaziali del sistema giuridico-religioso romano. Mundus, templum, urbs, ager, Latium, Italia, in ANRW, II, 16,1, 1978, p. $440-553$.

Coarelli 1981 = F. Coarelli, L'Area Sacra di Largo Argentina. Topografia e storia, in L'Area Sacra di Largo Argentina, I, in Studi e Materiali dei Musei e Monumenti Comunali di Roma, Roma, 1981, p. 9-51.

132. Le Gall 1976, p. 522; Torelli 1986, p. 169; Coarelli 1997, p. 216.
Coarelli 1997 = F. Coarelli, Il Campo Marzio, vol. I, Roma, 1997.

Coarelli $2003^{3}$ = F. Coarelli, Roma. Guida Archeologica, Roma-Bari, $2003^{3}$.

Comella 1986 = A. Comella, I materiali votivi di Falerii, Roma, 1986.

Corretti 1987 = A. Corretti, Civita Castellana, in BTCGI, V, 1987, p. 323-368.

Di Stefano Manzella 1990 = I. Di Stefano Manzella, Lo stato giuridico di Falerii Novi dalla fondazione al III secolo d. C., in Falisci, p. 341-368.

Dumézil 1954 = G. Dumézil, Iuno S. M. R., in Eranos, 52, 1954, p. 105-119.

Dumézil 1980 = G. Dumézil, Camillus : a study of IndoEuropean religion as Roman history, Berkeley, Los Angeles, 1980

Dumézil $2001^{2}=$ G. Dumézil, La religione romana arcaica, Milano, 2001².

Dury-Moayers, Renard $1981=$ G. Dury-Moayers, M. Renard, Aperçu critique de travaux relatifs au culte de Junon, in $A N R W$, II, 17,1, 1981, p. 142202.

Dury-Moyaers 1984 = G. Dury-Moyaers, Réflexions à propos de l'iconographie de Iuno Sospita, in R. AltheimStiehl, M. Rosenbach (a cura di), Beiträge zur altitalischen Geistesgeschichte, Festschrift G. Radke, Münster, 1984, p. 83-101.

Eisenhut 1963 = W. Eisenhut, Quiris, Quiritis, Curis, Cur(r)itis, in RE, XXIV, 1, 1963, p. 1324-1333.

Evans 1939 = E. C. Evans, The cults of the Sabine territory, in Papers and Monographs of the American Academy in Rome, New York, 1939.

Falisci = La civiltà dei Falisci. Atti del Convegno di studi etru- 
schi ed italici, Civita Castellana - Forte Sangallo, 28-31 maggio 1987, Firenze, 1990.

Ferri 2006 = G. Ferri, L'evocatio romana $-i$ problemi, in SMSR 72, n. s. XXX, 2, 2006, p. 205-244.

Ferri 2009 = G. Ferri, Voltumna-Vortumnus, in C. Braidotti, E. Dettori e E. Lanzillotta (a cura di), Studi in memoria di Roberto Pretagostini, II, Roma, 2009, p. 993-1009.

Ferri 2010a $=$ G. Ferri, Tutela segreta ed evocatio nel politeismo romano, Roma, 2010 (Mos Maiorum. Studi sulla tradizione romana, 4).

Ferri $2010 \mathrm{~b}=\mathrm{G}$. Ferri, Tutela urbis. Il significato e la concezione della divinità tutelare cittadina nella religione romana, Stoccarda, 2010 (Potsdamer Altertumswissenschaftliche Beiträge, 32).

Funke $1981=$ H. Funke, Götterbild, in Reallexikon für Antike und Christentum, XI, 1981, p. 659-828.

Giannelli 1942 = G. Giannelli, Iuno, in Dizionario epigrafico di antichità romane, IV, 1942, p. 211-240.

Giardina 1997 = A. Giardina, L'identità incompiuta dell'Italia romana, in Id., L'Italia romana. Storie di un'identità incompiuta, Roma-Bari, 1997, 3-116.

Girard 1989 = J.-L. Girard, Minerva Capta : entre Rome et Faleries, in Revue des Études Latines, 67, 1989, 163-169.

Gustafsson 2000 = G. Gustafsson, Evocatio deorum : historical and mythical interpretations of ritualised conquests in the expansion of ancient Rome, Uppsala, 2000.

Huschke 1970 = P. E. Huschke, Das alte römische Jahr und seine Tage, Wiesbaden, 1970 (rist. anast. dell'ed. del 1869).

Hülsen 1909 = C. Hülsen, Falerii, in RE, VI.2, 1909, col. 1969-1971.

Icard-Gianolio $2004=\mathrm{N}$. Icard-Gianolio, Rites et activités relatifs aux images de culte :VI. L'image cultuelle manifestation de prodiges, in Thesaurus cultus et rituum antiquorum, II, 2004, p. 463-471.

Koch 1960 = C. Koch, Bemerkungen zur römischen Quirinus-Kult, in Id., Religio. Studien zu Kult und Glauben der Römer, Norimberga, 1960, p. 17-39.

Köves-Zulauf 1993 = T. Köves-Zulauf, Minerva Capta eine gefangene Göttin?, in Grazer Beiträge. Zeitschrift für die klassische Altertumswissenschaft, suppl. V, 1993, p. 159-176.

Lanciani 1990-2007 = R. Lanciani, Forma Urbis Romae, Roma, 1990-2007.

La Rocca 1990 = E. La Rocca, Iuno, Lexikon iconographicum mythologiae classicae, V, 1, 1990, p. 814-856.

Le Gall 1976 = J. Le Gall, Evocatio, in Mélanges offerts à J. Heurgon. L'Italie préromaine et la Rome républicaine, Roma, 1976, p. 519-524.
Loreto 1989 = L. Loreto, Il conflitto romano-falisco del 241/240 a.C. e la politica romana degli anni successivi, MEFRA, 101, 2, 1989, p. 717-737.

Manacorda 1999 = D. Manacorda, Iuno Curitis, Lexicon topographicum urbis Romae, III, 1999, p. 121-122.

Martin 1987 = H. G. Martin, Römische Tempelkultbilder. Eine Archäologische Untersuchung zur späten Republik, Roma, 1987.

Meuli 1975 = K. Meuli, Die gefesselten Götter, in Gesammelte Schriften, II, Basilea-Stoccarda, 1975, p. 10351081.

Montanari 1990 = E. Montanari, Mito e storia nell'annalistica romana delle origini, Roma, 1990.

Montanari 1993 = E. Montanari, Evocatio, in G. Filoramo (diretto da), Dizionario delle religioni, Torino, 1993, p. 271.

Montanari 2008 = E. Montanari, Presentazione della collana Mos maiorum. Studi sulla tradizione romana, in Santi 2008, p. 9-12.

Mora 1994 = F. Mora, Arnobio e $i$ culti di mistero. Analisi storico-religiosa del V libro dell'Adversus Nationes', Roma, 1994.

Moscati $1990=$ P. Moscati, Nuove ricerche su Falerii Veteres, in Falisci, p. 141-171.

Musti $1999^{8}=$ D. Musti, Storia greca. Linee di sviluppo dall'età micenea all'età romana, Bari, $1999^{8}$.

Musti 2005 = D. Musti, Magna Grecia. Il quadro storico, Bari, 2005.

Opitz 2009 = R. Opitz, Integrating lidar and geophysical surveys at Falerii Novi and Falerii Veteres (Viterbo), Papers of the British School at Rome 77, 2009, p. 1-27 e 335-43.

Pallottino $1984^{7}=$ M. Pallottino, Etruscologia, Milano, $1984^{7}$.

Palmer $1970=$ R. E. A. Palmer, The archaic community of the Romans, Cambridge, 1970.

Palmer 1974 = R. E. A. Palmer, Roman religion and Roman empire. Five essays, Philadelphia, 1974.

Pasqualini 1996 = A. Pasqualini, I miti albani e l'origine delle feriae Latinae, in Ead. (a cura di), Alba Longa : mito storia archeologia. Atti dell'Incontro di studio, Roma - Albano Laziale 27-29 gennaio 1994, Roma, 1996, p. 217-253.

Pasqui 1887 = A. Pasqui, Civita Castellana (antica Faleria) - Avanzi di tempio etrusco scoperti in contrada "Celle», Notizie degli Scavi di Antichità. Atti della Accademia Nazionale dei Lincei, 1887, p. 92-100.

Preller 1978 = L. Preller, Römische Mythologie, New York, 1978 (rist. anast. dell'ed. del 1865).

Radke 1965 = G. Radke, Die Götter Altitaliens, Münster i. W., 1965 (Fontes et Commentationes 3). 
Radke 1981 = G. Radke, Quirinus. Eine kritische Überprüfung der Überlieferung und ein Versuch, in ANRW, II, 17, 1, 1981, p. 276-299.

Rasenna $=$ Rasenna. Storia e civiltà degli Etruschi, Milano, 1986.

Rüpke 1990 = J. Rüpke, Domi militiae. Die religiöse Konstruktion des Krieges in Rom, Stoccarda, 1990.

Sabbatucci 1988 = D. Sabbatucci, La religione di Roma antica : dal calendario festivo all'ordine cosmico, Milano, 1988.

Santi 2008 = C. Santi, Sacra facere. Aspetti della prassi ritualistica divinatoria nel mondo romano, Roma, 2008 (Mos Maiorum. Studi sulla tradizione romana, 1).

Schwenn 1920-1921 = F. Schwenn, Der Krieg in der griechischen Religion, in Archiv für Religionswissenschaft, 20, 1920-1921, p. 299-322.

Stopponi 1994 = S. Stopponi, Orvieto, in BTCGI, XIII, 1994, p. 1-88.

Torelli $1986=\mathrm{M}$. Torelli, La religione, in Rasenna, p. 159-237.

Van Doren 1954 = M. Van Doren, Peregrina sacra. Offizielle Kultübertragungen im alten Rom, in Historia, 3, 1954, p. 488-497.

Wissowa $1899=$ G. Wissowa, Capta, in RE, III,2, 1899, col. 1554

Wissowa $1912^{2}=$ G. Wissowa, Religion und Kultus der Römer, Monaco, 1912².
Ziehen 1949 = L. Ziehen, Palladion, in RE, XVIII,3, 1949, col. 171-189.

Zimmermann 1986 = J.-L. Zimmermann, La fin de Falerii Veteres : Un témoignage archéologique, in The J. Paul Getty Museum Journal, 14, 1986, p. 37-42.

Ziolkowski 1992 = A. Ziolkowski, The temples of MidRepublican Rome and their historical and topographical context, Roma, 1992.

Abbreviazioni

ANRW = Aufstieg und Niedergang der römischen Welt : Geschichte und Kultur Roms im Spiegel der neuen Forschung, ed. H. Hildegard Temporini e W. Haase, Berlino-New York, 1972 s.

BTCGI = Bibliografia topografica della colonizzazione greca in Italia e nelle Isole Tirreniche, dir. G. Nenci e G. Vallet, Pisa-Roma, 1977 s.

$C I L=$ Corpus Inscriptionum Latinarum, Berlino.

ILS = Inscriptiones latinae selectae, dir. H. Dessau, Berlino, 1892-1916.

$L T L=$ E. Forcellini, Lexikon Totius Latinitatis, Padova, 1864-1926.

SMSR = Studi e Materiali di Storia delle Religioni, Roma. $T L L=$ Thesaurus Linguae Latinae, Lipsia, $1900 \mathrm{~s}$. 


\section{Mélanges de l’École française de Rome}

ANTIQUITÉ

\section{Directeur}

Catherine VIRLOUVET

Directrice de l'École française de Rome

\section{Rédaction}

Yann RiviÈRE

Directeur des études pour l'Antiquité

Directeur des publications

Richard FIGUIER

Comité de lecture

Jean-Michel DAvid

Université de Paris I

Peter GARnsEy

Cambridge University

Jean-Pierre GuLHemBet

École normale supérieure, Lyon

Daniele MANACORDA

Università degli studi di Roma III

Marinella PAsquinucci

Università degli studi di Pisa

Gilles SAURON

Université de Paris IV 